(3)

Volume 21, 2018

\title{
SHIFTING PARADIGMS IN INFORMATION FLOW: AN OPEN SCIENCE FRAMEWORK (OSF) FOR KNOWLEDGE SHARING TEAMS
}

\author{
Megan Potterbusch* \\ Gaetano Lotrecchiano \\ * Corresponding author
}

George Washington University, Washington, DC, USA

George Washington University, Washington, DC, USA

mpotterbusch@email.gwu.edu

Glotrecc@gwu.edu

\begin{abstract}
Aim/Purpose This paper explores the implications of machine-mediated communication on human interaction in cross-disciplinary teams. The authors explore the relationships between Open Science Theory, its contributions to team science, and the opportunities and challenges associated with adopting open science principles.

Background Open Science Theory impacts many aspects of human interaction throughout the scholarly life cycle and can be seen in action through various technologies, which each typically touch only one such aspect. By serving multiple aspects of Open Science Theory at once, the Open Science Framework (OSF) serves as an exemplar technology. As such it illustrates how Open Science Theory can inform and expand cognitive and behavioral dynamics in teams at multiple levels in a single tool.
\end{abstract}

Method This concept paper provides a theoretical rationale for recommendations for exploring the connections between an open science paradigm and the dynamics of team communication. As such theory and evidence have been culled to initiate a synthesis of the nascent literature, current practice and theory.

Contribution This paper aims to illuminate the shared goals between open science and the study of teams by focusing on science team activities (data management, methods, algorithms, and outputs) as focal objects for further combined study.

This paper is one in a Special Series on Transdisciplinary Communication

Accepting Editor Shalini Misra | Received: October 15, 2017 | Revised: March 21, April 19, 2018 | Accepted: April 21, 2018.

Cite as: Potterbusch, M., \& Lotrecchiano, G. (2018). Shifting paradigms in information flow: An open science framework (OSF) for knowledge sharing teams. Informing Science: the International Journal of an Emerging Transdiscipline, 21, 179-199. https://doi.org/10.28945/4031

(CC BY-NC 4.0) This article is licensed to you under a Creative Commons Attribution-NonCommercial 4.0 International License. When you copy and redistribute this paper in full or in part, you need to provide proper attribution to it to ensure that others can later locate this work (and to ensure that others do not accuse you of plagiarism). You may (and we encourage you to) adapt, remix, transform, and build upon the material for any non-commercial purposes. This license does not permit you to use this material for commercial purposes. 
Findings

Recommendations for Practitioners

Recommendation for Researchers

Impact on Society

Future Research

Keywords
Team dynamics and characteristics that will affect successful human/machine assisted interactions through mediators of workflow culture, attitudes about ownership of knowledge, readiness to share openly, shifts from group-driven to user-driven functionality, group-organizing to self-organizing structures, and the development of trust as teams regulate between traditional and open science dissemination.

Participation in open science practices through machine-assisted technologies in team projects/scholarship should be encouraged.

The information provided highlights areas in need of further study in team science as well as new primary sources of material in the study of teams utilizing machine-assisted methods in their work.

As researchers take on more complex social problems, new technology and open science practices can complement the work of diverse stakeholders while also providing opportunities to broaden impact and intensify scholarly contributions.

Future investigation into the cognitive and behavioral research conducted with teams that employ machine-assisted technologies in their workflows would offer researchers the opportunity to understand better the relationships between intelligent machines and science teams' impacts on their communities as well as the necessary paradigmatic shifts inherent when utilizing these technologies. open science theory, research transparency technology, teams, open science framework

\section{INTRODUCTION}

In an effort to promote collaboration in research teams tackling complex problems, many new initiatives exist on local, regional, and national levels that aim to bring stakeholders together into crossdisciplinary teams so as to accelerate or diversify solutions. Many of these initiatives in the United States stem from responses to executive and federal mandates from agencies like the National Academies of Science (NAS), the National Institutes of Health (NIH), the National Science Foundation (NSF), private funders as well as executive orders that have required greater collaboration within and across disciplines requiring more team-oriented scientific approaches (Bennet, Gadlin, \& LevineFinley, 2010; National Research Council, 2015; Obama, 2015). As a result, team-initiated projects have increased over the past decade (Jones, Wuchty, \& Uzzi, 2008; Porter, Roessner, \& Heberger, 2008; Ranwala et al., 2017) and outputs from these diverse teams have shown a noticeable increase across fields and sectors to value team efforts in science (Bahney et al., 2016; Hinnant et al., 2012). Success measures of these cross-disciplinary teams continues to be mainly attributed to published scholarly outputs in an attempt to justify and maintain that cross-disciplinary teaming effectiveness can be observed through publications impact and diversity of authorship (Bales et al., 2014; Rosas, Kagan, Schouten, Slack, \& Trochim, 2011).

While efforts to justify increased impact of cross-disciplinary teams through bibliometric measurement methods like those mentioned above has provided insights into some of the advances made by team-initiated project teams, bibliometric analyses fall short in explaining the interactive dynamics of stakeholder involvement in scientific teams (Engwall \& Blockmans, 2014; W. Klein \& Bloom, 2005). A series of concerns about the measurement of contributions among scientific stakeholders emerges as sharing knowledge advances with our new and more open-centric technologies that defy the confines of traditional publishing avenues. These concerns include issues around recognition of author contribution; new forms of publishable research materials; ownership of knowledge; team contributions over the lifespan of a project; non-author contributions; and the impact that stakeholders have 
on the direction of research and scholarly projects. These along with other issues beg the question: How do cross-disciplinary teams leverage technology in order to document, provide access to, and preserve scientific contributions?

Embedded within the research and publication process is a complex array of interactions that, until the advent of communication and research management technologies, remained relegated to conversation, physical lab books, written notes, and even casual outings amongst colleagues each of which goes unanalyzed or critiqued for their contributions to the process of science. With the rise of technologies that support, capture, and document much of the activity once veiled by these frequently inaccessible artifacts, the possibility emerges for much of the interactive material involved in scientific activity to be exposed and shared. This allows for scientific interactions and the 'process' of science to be part of the dissemination of scholarly activity. Thus, both products — such as data, methods, and algorithms - and the communication processes embedded in the act of doing research can become objects of study. The questions available for scientific inquiry multiply exponentially. Data could become part of a meta-study. Computational algorithms could be built on in order to answer larger-scale questions. The communication processes of teams could be assessed to better understand the elements that go into team effectiveness and impact, which in turn would allow for the implementation of enhanced practices based on effective use of research assisting technology.

However, when it comes to making research results available to the public, the typical scholarly communication workflow remains very traditional, closely following an age old path; one that starts with the submission of an initial article draft to a publisher or editor, follows through private peer review, and frequently ends in a single form of final output: an article published in a scholarly journal. This process remains the gold standard for information flow from scientists to communities of others scientists to the mainstream public knowledge base (Weimer \& Andrew, 2013). The dissemination of scientific material that relies solely on this publication model neglects and loses out on the many other research products outlined above while simultaneously driving many of the behaviors of scientists wanting to attain the gold standard of publishable results - generally a citation, measured in bibliometric assessment. Several issues become easily apparent: from the reluctance to share openly about the complex processes and collaborator negotiations occurring in the course of research activity, to the lack of transparency and trust as it applies to scholarly recognition and credit, and to the still limited sharing of secondary research artifacts often not included in final publication outputs. All of these scientific teaming artifacts are veiled from the consumer of the science, thus, making the evaluation of how successful teams work effectively a mystery to science stakeholders and consumers. Many of these interactions, and others, that typically occur within science teams, have the potential to provide rich data, which could greatly increase our understanding of team high-effectiveness processes.

By using tools and technology that now exist and progressing away from the traditional flow of information, research teams can adapt to both the mandate and modern trends, which in turn can fundamentally inform interdisciplinary team science. This transition, in combination with increased transparency, would allow team science researchers to understand teaming processes and expand possible research about teams. Informal scientific contributions and communications are now increasingly captured, because professionals now communicate and collaborate more often than ever through written virtual formats (Google Docs, Dropbox, emails, tweets, blogs, etc.) that serve as artifacts of interaction. Digital platforms bridge multiple stakeholders representing different disciplines, but more than that, they provide venues that facilitate learning and knowledge integration which are critical to the advancement of cross-disciplinary team science initiatives (Pennington, 2011). These written digests and digital records of information serve as important data sources by which to map the interactions that humans have with one another as individuals while they collaborate especially in scientific teams. In an era when technological communications are increasingly more freely available across scientific classes and the ability of individuals to access these technologies is only limited by one's freedom to explore them, further consideration and study of how communication technologies, 
especially in scientific sharing and collaborative activities, contribute to the advancement of science to achieve new levels of innovation is needed.

This paper will explore how an understanding of human communication in cross-disciplinary teams might be enhanced through open science based technologies. In addition to reviewing the problems associated with cross-disciplinary communication through collaboration technologies, the authors will explore Open Science Theory (OST) as a framework from which to consider the value of machine-assisted collaboration to enhance project team knowledge mapping and interactions. As a case example, the Open Science Framework (OSF), a tool that supports collaborator controlled workflow transparency, will be introduced as a platform by which certain key issues associated with crossdisciplinary team engagement might be understood and improved.

\section{Clarification of CONCEPTS}

In order to effectively discuss this topic, a few terms require clarification. For the purpose of this paper, the term "communication" will be used for traditional forms of exchange such as direct messages, peer review, and any other form of conversation (i.e., reciprocal communication) (Rollman, Krug, \& Parente, 2004). More frequently used in this article is "interaction," which includes all forms of "communication" (verbal and behavioral), as well as newer forms of interaction such as reviewing workflow materials, exploring interactive visualizations, or copying and building off someone's idea (Parasuraman, Sheridan, \& Wickens, 2000).

"Team" indicates collaborators that seek to solve a problem or work on a given research project or grant (Wuchty, Jones, \& Uzzi, 2007). "Team science" refers to the act of addressing scientific problems as a group dedicated to shared scholarly goals (Stokols, Hall, Taylor, \& Moser, 2008). "Stakeholders" are invested members of the teams' scholarly community who may serve in the capacity of editorial reviewers or as participants in the team's professional network. Further removed for the core of scientific teams and their leadership are "consumers" who are recipients of research products but do not typically communicate directly with the team and their stakeholders. This group may participate through contribution in a very distant capacity. Depending on the direct impact of the research or scholarly activity on consumers, they may also be stakeholders, but generally they do not interact in a direct way with teams of scientists (Gibson, 2000).

"Open science" is generally understood as a variety of activities promoting transparency within research, motivated by many beliefs or core values. As the EU-funded project FOSTER Plus (2016) defines it, "Open Science is the practice of science in such a way that others can collaborate and contribute, where research data, lab notes and other research processes are freely available, under terms that enable reuse, redistribution and reproduction of the research and its underlying data and methods." Peter Suber (2012) described open science as "combining [open access] OA texts, open data, and open-source software, and providing these sorts of openness at every stage of a research project, not just at the end in reporting results" (p. xi). For a better understanding of the beliefs or motivations behind adopting, or not adopting, open science practices, one must look to the nascent field of Open Science Theory (OST).

As outlined by Fecher and Friesike (2014), OST is best conceptualized in its entirety by highlighting the multiple perspectives or schools of thought, which motivate advocates, supporters, and practitioners of the activities outlined above. A common supporting theory of open science is the desire to democratize knowledge by lowering financial and other access barriers and reducing copyright restrictions to science in general and data or publications in particular (Fecher \& Friesike, 2014). Similarly, some open science advocates and practitioners work to help make science more intellectually accessible and engaging to the public through more opportunities for general involvement in the process of science and through blogging/micro-blogging. Open science generally includes the importance of alternative forms of measurements (i.e. altmetrics, credit for peer review, etc.) and the infrastructure involved in supporting efficient, collaborative, and interoperable platforms and tools, 
because new forms of contribution require new forms of measurement in order to be acknowledged (Fecher \& Friesike, 2014; Priem, Taraborelli, Groth, \& Neylon, 2010). A pragmatic motivation drives many open science practitioners: the belief that "knowledge-creation could be more efficient if scientists worked together" and that this can be accomplished by "opening up the process of knowledge creation" (Fecher \& Friesike, 2014, p. 20). Each perspective or motivation for open science can be viewed and explored somewhat independently of others, but the activities involved, such as sharing or re-using open data, overlap across these different schools of thought.

In order to focus on the impacts and implications of open science on team dynamics, the discussion of OST here focuses primarily on the pragmatic school, which includes transparency in the process of knowledge creation, and the infrastructure school, which pertains to open platforms, tools and services (Fecher \& Friesike, 2014). The goal behind this exploration of OST is to better understand the ways that it allows for new forms of interaction at all levels: from team communications, such as collaborative document writing, to stakeholder and consumer interactions, such as exploring published data using author/publisher provided visualization tools.

In many ways open science is a radical expansion of the historical forms of scientific dissemination (e.g., published articles, scientific conferences, and poster presentations). As opposed to only sharing final, published articles, open science allows researchers to provide more access points to their work. As such, in the context of this article, "publication" can refer to "any product (e.g., publications, datasets, experiments, software, web sites, blogs) resulting from a research activity, that is relevant to the interpretation, evaluation, and reuse of the activity or part of it" (Assante, Candela, Castelli, Manghi, \& Pagano, 2015), which is accessible to the public. Accessibility in this case includes preservation and dissemination activities such as depositing the materials for publication into a repository that provides a Digital Object Identifier (DOI) or other form of persistent, interoperable, unique identifier (International DOI Foundation, n.d.).

\section{TOOLS AND TECHNOLOGY IN OPEN SCIENCE AND TEAM SCIENCE}

Many modern tools and technologies that support research workflow and communications fall on a continuum from opaque, or "closed" to transparent or "open." Tools and technology for in-team communication include anything from email to conference-call software and from project management software to shared cloud storage. These communication tools can be designed for privacy or openness. In fact, many tools support either type of use. For example, a team can use Google Hangouts for a private meeting or choose to use Hangouts On Air which broadcasts live to YouTube.com in order to include stakeholders in the conversation. Teams can also choose to privately share documents using a cloud storage provider like Dropbox or publically share a link to a folder in order to promote stakeholder access to working documents and provide a view into the evolution of their research. Similarly, tools and technology specifically designed for communicating with stakeholders and the general public can be more or less open. In this case, traditional publication is the most "closed" or limited-access communication system for the research process, because the only part of the research traditionally available is the completed manuscript. This process is becoming more aligned with open science principles through the increasing distribution of preprints, and availability of open data sets, software, and other supplementary materials. These tools and technologies can be visualized along an intersecting continuum (Figure 1). The point of intersection comes in the form of open workflow technology; in other words, tools designed for both the active work of research and the open publication of materials. At this time, few tools exist that wholly encompass both parts of this communication continuum, and only the Open Science Framework (OSF), created by the Center for Open Science and designed "to provide free and open source project management support for researchers across the entire research lifecycle" (Center for Open Science, n.d.-b), exemplifies many of the principles of open science outlined above. 


\section{Transparent workflow and communications}

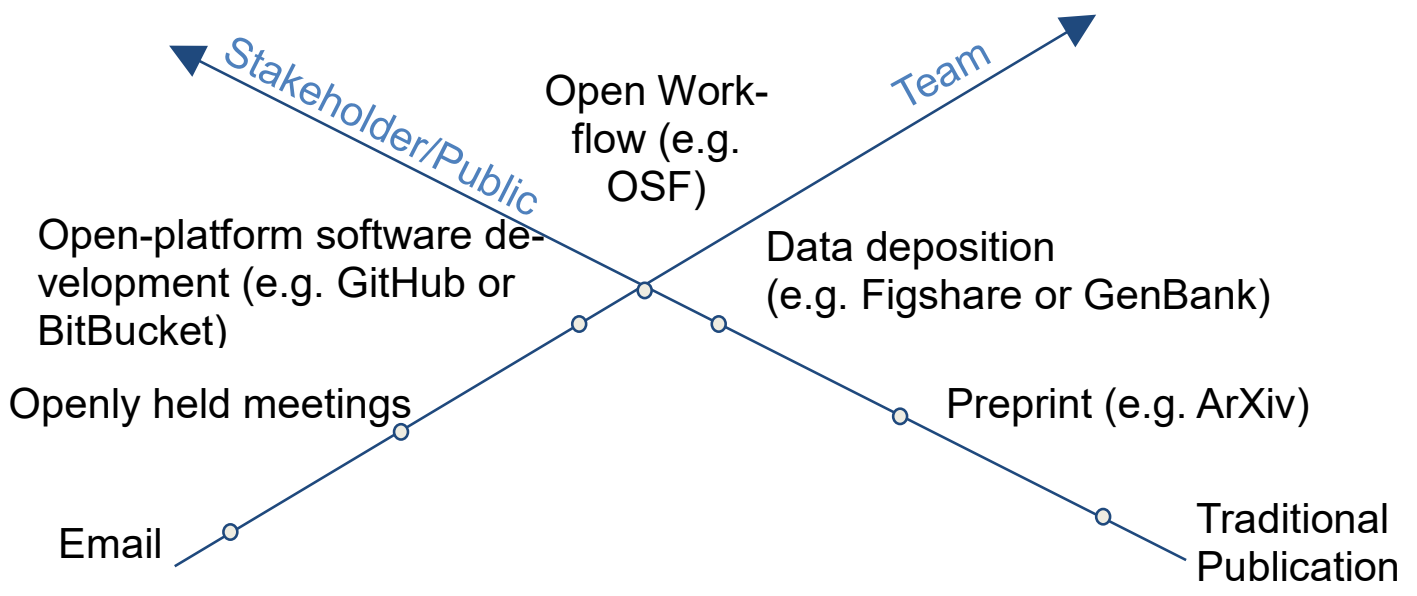

\section{Opaque workflow and communications}

Figure 1. Technology Continuum: from Opaque to Transparent Communication within Teams and with Stakeholders

A published OSF project that has been used as an active workspace throughout a research project allows for transparency of in-team research activities and public communication with stakeholders. This workspace would contain the version history, commentary, and other activities that contributors have carried out throughout the project while simultaneously providing access to the final dataset, code, and/or any other supplementary materials created in the course of normal research (e.g., codebooks and methodology). Publication of an OSF project can be achieved via making a project public and generating an indexed, permanent identifier (e.g., a Digital Object Identifier or DOI see http://www.doi.org/) or by generating a registration, which is a snapshot of a project at a particular moment in time (e.g., https://osf.io/aurjt/). These outputs and the activity feed generated over the course of normal OSF use create an opportunity to better understand the types of interactions occurring within team research activities. Coding of the individual, team, and even stakeholder interactions becomes possible when and if a project becomes public. This increases workflow transparency within and across teams with similar interests and with a stakeholder and consumer invested in the evolution of the science. It provides access to an otherwise untraceable exchange of knowledge and social relationship development in science, which is not only important to the study of teams but also important to maintaining both individual autonomy and team cohesion. If the suggested workflow is followed, certain registrations can even be published as Registered Reports in journals such as eLife, which provides teams conducting research with an incentive and an opportunity to share their work at different stages and in different ways (Center for Open Science, n.d.-a). Another form of workflow transparency can be achieved through the use and open sharing of Electronic Lab Notebooks; although these tools are not publishable in the same way, sections often can be issued DOIs and be made publically available and whole notebooks can be downloaded to PDF for publication, individual-sharing, and preservation (LabArchives, 2018). For teams that work primarily within software development, version control tools such as GitHub can also publish their entire workflows openly by connecting their repository (i.e., workspace) to a preservation platform that issues DOIs such as FigShare or Zenodo. Although not all communications or supplementary materials are likely to be attached to the software, this is still a way of publishing a workspace rich with team interactions. 
Although open, transparent workflow - as a radically transparent approach to open science - promises the most disruption and the most potential benefits from openness, it can also present great risks (Scheliga \& Friesike, 2014), such as revealing personal file management habits via activity tracking and decreasing potential competitive advantage through shared datasets and software pipelines. Thus, there are other aspects and tools within this continuum to be addressed. Since the assumption underlying the infrastructure school of thought is that "efficient research depends on the available tools and applications" (Fecher \& Friesike, 2014, p. 20), considering the various related technologies facilitates an understanding of the additional types and forms of interaction that have become possible in recent years. For example, open infrastructure technology for team communication includes such resources as Authorea (https://www.authorea.com), a collaborative writing tool that facilitates publication through templates and publisher integrations while also supporting embedding data and code into figures with embedded data and code, and GitLab (https://about.gitlab.com), an open source software development and code sharing resource. These and other collaboration oriented technologies allow researchers to do more of their work openly or to facilitate lower barriers to making their research transparent in strategic ways.

As previously mentioned, many new forms of machine-mediated communication have arisen lately. This includes sharing research building-blocks such as scientific algorithms, raw data, processed data, and pre-published articles. New funder and publisher requirements regarding sharing data associated with research and publications are responsible for some of the changes towards increased sharing of supplementary materials. Open data files may, but do not necessarily, include details describing collection and/or processing methodology or associated algorithms. Similarly, not all researchers who choose to share the code or algorithms that underpin their scientific discoveries choose to include their version history, associated data sets, or complete workspace with their shared software. Instead, algorithms may be shared as a single final output in one of the previously mentioned repositories, on personal websites, or through software papers. One website identifies and indexes software resources for the astronomy community is the Astronomy Source Code Library (http://ascl.net/). These are all pieces of software that have been cited in published articles and also made available via NASA ADS (https://ui.adsabs.harvard.edu/); thus, making the code accessible in the same index as other scholarly resources - predominantly articles.

Data repositories, such as GenBank (https://www.ncbi.nlm.nih.gov/genbank), house data collected from many researchers and provided in highly specific formats with particular descriptions (metadata) that facilitate discovery of data sets from many entry points. By increasing distribution, sharing data in this way facilitates discovery and allows additional stakeholders to explore areas for novel research. In the case of GenBank, deposited data are exchanged on a daily basis across the "International Nucleotide Sequence Database Collaboration, which comprises the DNA DataBank of Japan (DDBJ), the European Nucleotide Archive (ENA), and GenBank at NCBI" (Johnson, 2017). Internationally broad distribution increases access for researchers, which means that data collected by a single research team can become even more impactful to the scientific community overall. Data repositories are not limited any particular scientific field for example, "ICPSR [Inter-university Consortium for Political and Social Research] maintains a data archive of more than 250,000 files of research in the social and behavioral sciences" (Inter-university Consortium for Political and Social Research, n.d.) and FigShare provides a domain agnostic digital repository for researchers interested in preserving and receiving credit for all their research outputs (including data and software code). With both discovery and citation of data facilitated by the systems, authors and data collectors can receive additional scholarly credit for their contributions as well; thus, providing personal incentive to share beyond simply compliance with a government mandate (Alicea, 2016, Holdren, 2013).

Possible stakeholder communications have also become more open through such platforms as preprint servers and data repositories. Although ArXiv (https://arxiv.org) has existed for decades, supporting the disciplines of physics, astronomy, and math, preprint servers like PsyArXiv (a preprint server for psychology) and SocArXiv (a preprint repository for social sciences) are becoming increas- 
ingly popular places for scholars to make a pre-publication version of their soon-to-be-published articles available ("ArXives of Earth science," 2018; Fister, 2016). Allowing researchers to read and share cutting edge research early, preprint servers also facilitate public access to articles thus allowing for broader engagement with the material and increased visibility for the work and the research team (Berg et al., 2016). As much as these resources do disrupt the traditional scholarly communications landscape, it should be understood that they do not increase the openness or transparency of workflows or other interactions within a research team. Thus, pre-prints and open data resources provide expanded community access and consumption without necessarily impacting or highlighting team dynamics.

Visualization tools are not inherently part of open science. However, when they are used to create embedded interactive visualizations that allow consumers to better understand the published research and especially when they are nuanced or complex enough for consumers to make discoveries for themselves, the visualization then exemplifies the Public School of Open Science Theory as outlined by Fecher and Friesike (2014). In fact, it can be argued that good information visualization in general should facilitate user interaction and thus deeper meaning making on the part of consumers (Kosara, Hauser, \& Gresh, 2003). Additionally, when code such as R (an open source coding language) is used to process data and generate figures, these figures can be published (by supporting platforms) with the data and code embedded and automatically generating the figure, which allows for increased scientific transparency. Inferring from open source software development communities, who rely on openness, this additional transparency allows for increased intellectual interaction between the information consumer and the research group who wrote the article, because the consumer can examine the process that went into creating the visualization, which in turn allows for a clearer understanding of the work (Dabbish, Stuart, Tsay, \& Herbsleb, 2012).

\section{OPEN WORKFLOW TECHNOLOGY IN TEAM SCHOLARSHIP}

Supporting the diverse needs, professional and interpersonal, of team members within open technology requires flexibility either within the technology itself or in how the teams apply the technology within their workflows. The OSF platform accommodates this need for flexibility by allowing for different levels of openness and customizable structure. Teams desiring to implement a fully open workflow may still keep their project private until they are ready to share it. Conversely, teams not wishing to publish their workflow may still utilize the tool to provide open access to specific parts of their finished projects by uploading finalized files upon completion of their research. Researchers may also connect their preferred workflow tools, such as Google Drive or Dropbox, to the platform and allow their colleagues to engage in the OSF without them while still providing common team access to their materials. Adaptability of structure and terminology allow interdisciplinary teams to define the structural and descriptive vocabularies appropriate for their research needs and not be defined by a single discipline or by the platform of choice. Thus, teams are able organize their OSF projects to best suit specific team needs while also facilitating future public access to some or all of their work.

Consumers and stakeholders can build on the work of others without changing it and while maintaining attribution and provenance intrinsically within the OSF - through a process called forking. As potentially beneficial and timesaving this functionality can be for the scientific ecosystem in general, concerns arise for many researchers about open sharing of materials and the possibility that their research project could be forked may increase this apprehension (Scheliga \& Friesike, 2014). To assure that intellectual credit remains with the original research group even when the project is public, the OSF provides citation guidance on every project that is created, and allows the administrators of the project to create DOIs, which are recognized throughout the publication system as a mark of a permanently available resource, as previously mentioned. Researchers can further develop scholarship by making processes (e.g., workflow and previous versions of files) as well as products (i.e., data sets, software, or protocols) public, shareable, and citable. Increasing the social value for researchers shar- 
ing materials and for researchers studying or otherwise learning from openly shared materials is necessary for these functions to be valued and used (Alicea, 2016).

Naturally, different teams will need different tools and will be interested in different levels of openness. One example of a research group's use of the OSF is the Eleanor Roosevelt Papers Project. This is a historical documentation project centering on selection, transcription, and annotation of primary source materials (Brick \& Regenhardt, n.d.). Seeking project management and communication technology, an editor and decision maker for this group reviewed their team needs as well as the functionality provided by the OSF. Requiring free storage, version control, automatic tracking of user activity, and different levels of access control, among other functions, the editor selected this tool for the project. The ability to make the platform public remains low on the research team's list of desires or motivations for adoption, because they have other methods for digital publication. However, now that the team's project is entirely in one location and organized, the editors are empowered to publish their workflow and work space within the OSF in the future with ease. Since their use of the technology is both novel and exemplary, their workspace could serve as an inspiration and template for other digital humanists or historians as well as being a potential object of study for team scientist interested in studying team dynamics or workflow behavior. External to the primary research, this team's implementation of the OSF has sparked curiosity from various stakeholders including fellow humanities researchers, librarians, and funders as demonstrated by numerous private and public speaking engagements on this novel implementation of the tool and on their visible and demonstrable workflow.

Another tangible example of a multi-level team working together and exploring judicious and appropriate use of open tools in a workflow is the University Seminar Creating a Culture of Collaboration at George Washington University (C3@GWU). Made up of "knowledge communities ("think tanks") [that] represent GWU, regional institutes, and federal government stakeholders" (https://blogs.gwu.edu/collaborativeculture/welcome-to-c3gwu/), C3@GWU engages stakeholders in trans-, inter-, and cross- disciplinary studies at different levels. This group uses fundamental technologies for communication such as email and teleconferencing services as well as a shared Google Drive for both administrative documentation and meeting notes, and a blog for external communication of activities and events performed or hosted by C3@GWU. By using the blog and making their work easily accessible to anyone, this group participates in open science in accordance with the "public school" of thinking. Through the comment feature, information consumers and stakeholders have a space to interact with the research team directly.

From an interest in exploring further opportunities for bi-directional non-team interactions and other aspects of open science in order to accomplish their own goals, C3@GWU reviewed their current abilities and what they wished to do, and found that there were additional levels of interaction available via Open Science Theory inspired tools and technology. Through the communication tools outlined above, this group can be centrally organized around internally defined aims and goals; however, they could explore open workflow tools like the OSF, which can be used to facilitate non-team member commenting and contributions on public projects. These new forms of interaction could allow stakeholders to become agenda drivers and allow them to communicate in dialog with researchers.

As is the hope of the Public School of Open Science Theory, the openness of the platform (no pay subscription, discoverable through normal search engine searches) creates both opportunities for discovery by new consumers and additional space for interactions and engagement beyond one-way communication. Scholarship conducted publicly can lead to reservations on the part of researchers for many reasons, and, thus, selecting a single tool that facilitates both open (public) communication and private communication can help. In this case, the ability to provide public (seminar/stakeholder) and private (team-only) sections within a single workspace, such as the OSF provides, can help to mitigate the risks/challenges inherent in working entirely in the open. 
Although exploring aspects of OST intrigues many team members of C3@GWU, overall the active needs of the team are generally being met by the other technologies that are already in place. Thus, most think tanks, or smaller teams within the larger research group, have not adopted the OSF. The teams that have been using the tool did so when interest in exploring open science technology intersected with a need to communicate bi-directionally with stakeholders at a conference. Since this public use occurred within the initially developed project structure, additional, future uses by other think tanks will be intellectually tied to this public component. This intellectual and technological connection will allow future consumers to better understand the relationships between the think tanks without overall adoption of the tool happening simultaneously (or ever necessarily).

There are notable differences however between working privately in a tool not designed for open science (e.g., email) and working privately in the OSF. By design, the OSF can be published along with all of the communications and interactions that took place in the course of research. With some notable exceptions (e.g., political figures), private email communications do not hold the same promise of future transparency or publication that working and communicating in a platform with built in publication capabilities; thus, the structural and content decisions must be carefully considered. Depending on decisions made by the team regarding how they would like to prepare their workspace for publication, their registration could even be published as a preprint (Assante et al., 2015). Projects that are published as registrations can continue to serve as active workspaces for the current team without impacting the contents of the registration. If the project is made public at the same time that the registration is created, even more possible interactions for consumers and stakeholders are generated. Suddenly there are two forms of available research (with different citations) that can be reviewed and understood. Stakeholders can even interact with research by duplicating or "forking" the public project in order to build on the research in a new direction without losing the record of the original creator/author/contributor/team's work (and thus a record of the original intellectual contribution is maintained for measuring impact and credit). Analytics for OSF projects include the number of times a project has been "forked" or used as a template along with more standard altmetrics (e.g., downloads, views, site visits, etc.) (Priem et al., 2010). This introduces a new form of contribution to the scholarly discourse, much like a teacher who molded a student's understanding of a concept or their way of shaping their research, building additional research off a project fork allows a consumer of the secondary researcher's work to clearly understand where they started from and who influenced them or provided them with the fundamental resources to build a new branch onto the original research.

However, in some cases, the ability to publish the workspace creates too much of a risk for the potential user - risks such as scooping or compromised intellectual property rights - which outweighs any current, tangible benefits to increased transparency (e.g., increased reproducibility and understanding). Such is the case with a Think Tank within C3@GWU. Without a strong enough need or perceived benefit to outweigh the researchers' concerns, this team choose not to adopt the OSF. Instead their communication needs are satisfied with the other technologies employed by the project as a whole, and the space remains available if they choose to create final products to share without impacting the other Think Tank's flexibility and autonomy within the platform.

\section{OPEN SCIENCE READINESS IN TEAMS AND THEIR STAKEHOLDER COMMUNITIES}

Open science generally serves as a framework for conceptualizing a new form of information sharing and collaborative effort that brings with it important shifts to a number of notable areas within scientific teaming. Joint scientific and scholarly collaborations, the integration of knowledge and the stakeholder communities that are impacted, when utilizing an open science approach, can serve as nodes of information sharing and scientific advancement with greater impact (as measured by citations) than more traditional venues might normally yield (Evans \& Reimer, 2009; Gargouri et al., 2010). Thus far we have focused on the technological tools and the techniques that these tools add to 
a culture of shared and integrated knowledge economies. Such fluid and collaboratively based economies, those found in transdisciplinary endeavors, even with their high-functioning potential for collaboration across networks still pose threats to individuals, their traditional communities of science and practice, and the teams that generate and disseminate knowledge. Many opportunities arise by utilizing machine assisted communication but challenges also exist that require cognitive and behavioral consideration (Scheliga \& Friesike, 2014). Though these shifts are numerous and not yet fully considered, here we offer a few openings that are clearly apparent in the emerging open science culture suggesting a spectrum of change as collaborative technologies become more commonly utilized by teams and stakeholders within these knowledge economies (Hendriks, 1999).

\section{WORKFLOW CULTURE}

As has been stated, the traditional workflow environment in knowledge generation, the development of disseminable products through peer review, publication, and evaluation continues to evolve. In an age of machine-assisted technologies, parameters change so that in addition to increased speed and greater access to information, processes, and workflows also change requiring adjustments to otherwise time-tested processes of sharing and distributing information. Scientists and scholars are faced more than ever with challenges that test their decision making, behaviors, and strategies for maintaining individual, team, and stakeholder relationships in scientific projects. A culture that can embrace what open machine-assistance has to offer in securing unhampered knowledge generation and dissemination across boundaries will require the cultivation of cultures that intentionally introduce, maintain, and nurture continual and practiced open science and communication (Nosek et al., 2015).

Workflows that assume linear processes of knowledge generation - the progression from problem identification, data acquisition, and experimentation leading to publication - are now being challenged by other workflows that are more complex and iterative (Spiegler, 2003), assuming and accommodating for different partners with their differing investments, styles, and motivations for accessing and sharing knowledge (Gravani, 2005). The demand for more intelligent and intuitive information technologies grows as disparate communities strive to flatten the divides of geographies, time zones, language, and class often found across networks of knowledge generators (Haythornthwaite, Lunsford, Bowker, \& Bruce, 2006). However, technological advances that help to minimize these types of collaboration barriers are only part of the culture equation. Stakeholder and team member congruence, task interdependence, team commitment and participative decisionmaking need to be continually nurtured within a collaborative culture if open science is to be valued as a viable context in which to advance how knowledge is shared and disseminated in a new age of information fluidity (Lin, Shih, \& Sher, 2007). This cultural shifting within science communities draws our attention to how open science, technologies, and the new relationships these contracts promote, affect existing and emerging teams and stakeholder groups.

Knowledge producing scientific teams are similar to other types of organizations in that they ultimately strive to meet their pre-established goals. Ultimately, the goal of any science team is to generate knowledge around specified problems so as to impact the community and world that values the problem. Organizational culture, in this case team and stakeholder culture, plays an important and critical part in the achievement of these goals, as it has the ability to nurture or stifle processes that impact success. Numerous features inherent to team engagement can serve as challenges to maintaining a culture of high-functioning sharing of knowledge. These can include high diversity of membership (Kozlowski, Watola, Jensen, Kim, \& Botero, 2009), large team size (Tannenbaum, Mathieu, Salas, \& Cohen, 2012), designing deep integration of knowledge (Drath et al., 2008), navigating goal misalignments (Salazar, Lant, Fiore, \& Salas, 2012), permeating team and stakeholder boundaries (Hall et al., 2012), geographic dispersion, and securing high task interdependence (Burke et al., 2006).

Certain cultural behaviors are required to secure that information and workflow dynamics will foster integrated outcomes and counteract barriers to team science. These include (1) pairing the need for success with teaming procedures that complement these goals, (2) practicing the sharing of 
knowledge as a common team and stakeholder activity, (3) linking knowledge to identified and deeply shared values (for both teams and stakeholders) using the language of these values as a means to communicate results and impact, (4) constantly underscoring that information networks are human networks requiring a sensitivity about how these networks operate and need to be maintained, and (5) as in business corporate communities, recruiting scientific team members and stakeholders that already understand the value of openness and sharing so that team workflows can be reinforced through expansion and not hampered by each new addition to the network (McDermott \& O'Dell, 2001). Such organizational culture dynamics and behaviors when utilized within scientific teams ideally should be embraced by all stakeholders; however, strong leadership that reinforces that these dynamics has been proven to result is greater and more successful collaborative outcomes (Lin et al., 2007; Srivastava, Bartol, \& Locke, 2006).

\section{ATTITUDES ABOUT OWNERSHIP OF KNOWLEDGE}

Maintaining cultures of collaboration and open sharing of knowledge depend greatly on individual attitudes so that team members and stakeholders can adequately contribute to the organizational culture that will breed constant and consistent sharing of information. That collaboration is increasingly a requirement of scientific teams often put forth by funders and other external factors is not enough to ensure that such priorities are met without resistance by individuals within teams and across stakeholder groups even when individuals desire to enhance collaborative relationships (Hower, 2012). Individual preoccupations about ownership of contributions and insecurities about how such shared knowledge will be integrated into team products, recognized within one's own community of practice or science or even their academic home, and shared with a road audience of consumers often hamper the sharing of knowledge (Bock, Zmud, Kim, \& Lee, 2005). These internal conflicts can impede one's ability to adequately share information, trust a team or network, and even hamper the advancement of a science program especially if knowledge is withheld as a result of such insecurities (Lotrecchiano et al., 2016). Loss aversion (Fox \& Faver, 1984; Georghiou, 1988; Sonnenwald, 2007), lack of recognition and reward (Dasgupta \& David, 1994; Turpin \& Garrett-Jones, 2010), concerns about achieving promotion and tenure (Carayol \& Thi, 2005; Coleman, 1986; Harris, Lyon, \& Clarke, 2009; Horlick-Jones \& Sime, 2004; Maglaughlin \& Sonnenwald, 2005; Rhoten \& Parker, 2004; Zucker, 2012), and authorship embattlements (Barrett, Funk, \& Macrina, 2005; Lewis, Ross, \& Holden, 2012; Stokols et al., 2008) are just a few of the issues that can ensue from within a scientific team that struggles with diverse attitudes about knowledge sharing.

The challenges that can arise from inequitable attitudes about ownership of knowledge and more importantly the defining of what it means to share that which one also desires to own, concern teams that strive to advance science through collaborative effort. As teams grapple with these dynamics, team productivity and freedom to disseminate are not the only concerns. Rather as Rechberg and Syed (2013) have shown, these tensions can also impede the performance of individuals themselves within these networks as they grapple with moral and ethical dilemmas that are a part of negotiating what one might consider individual ownership and corporate ownership of contributions. In these instances, the authors recommend moving such issues to a level of moral consideration where agreements and contracts include ethical constructs and procedures that establish and increase trust, values, and fairness as knowledge management protocols (Rechberg \& Syed, 2013). These types of 'pre-nuptial' agreements are commonplace in many team science arrangements that ensure that such considerations are dealt with early on in the teaming process (Bennet et al., 2010).

\section{READINESS TO SHARE OPENLY}

Inviting participants to engage in scientific collaborations requires careful consideration of how team members and stakeholders will ultimately benefit from the efforts of a program of science. The invitation process may require leaders and teams to carefully weigh the scientific expertise of a potential collaborator along with the soft skills necessary for them to be an active and freely sharing member 
of a team. This is not always easy to negotiate, as it is not likely that all candidates with the appropriate scientific knowledge for a given research team will have the requisite soft skills needed to participate in a free sharing culture.

Readiness to collaborate in scientific teams is not a new concern to the science-of-team-science community who study team behaviors. Researchers developing measures of readiness have utilized many techniques to study multiple variables associated with this problem (Armstrong \& JacksonSmith, 2013; Lotrecchiano et al., 2016; Misra, Stokols, \& Cheng, 2015; National Research Council, 2015; Olson \& Olson, 2000). An individual's readiness to collaborate or one's collaborative orientation is often attributed to competency and leadership training that is (or should be) part of one's overall scientific training (Hall, Feng, Moser, Stokols, \& Taylor, 2008; Hoffman et al., 2013; C. Klein, DeRouin, \& Salas, 2006; Stokols, 2014) However, as is often more so the case, readiness to collaborate in teams is an iterative process that over time provides individuals opportunities and the vehicles by which to learn the necessary skills to adequately and successfully participate in the sharing of knowledge within teams. Research associated with technological readiness is also a factor. Technological readiness and, maybe more importantly, technological acceptance is particularly pivotal to issues of communication in these teams as each ultimately contributes to the degree to which scientific teams can more forward capitalizing on the benefits of machine assisted scholarship and communication tools that advance impact and dialogue with networks of scientists and stakeholders (Lin et al., 2007; Olson \& Olson, 2000).

Within open science landscapes, this ability to operate at level participation and sharing depends greatly on the requisite skills and one might consider appropriate readiness to be successful. In fact, goal setting measures of success that include a team's ability to integrate and generate synthesized knowledge may require teams of relatively technologically skilled members that can consistently work within a high-sharing and transparent environment. Even in proprietary environments where sharing of knowledge with consumers before a product is fully developed is unlikely, teams and networks of contributors are expected to share internally and their behaviors to do so are part of the production equation (Hansen \& Avital, 2005). Quality is also often measured by personalities (optimism, innovativeness, discomfort, and insecurity), which determine collaborative behaviors and in turn affect willingness and readiness to accept new and novel technological tools (Liljander, Gillberg, Gummerus, \& van Riel, 2006; Walczuch, Lemmink, \& Streukens, 2007). These personality traits can also be considered in the intrapersonal motivations a team member might have with regards to what drives their willingness to share knowledge (Andriessen, 2006; Mallinson et al., 2016; Swift, Balkin, \& Matusik, 2010). Readiness in collaborative teams will require that individual members can adequately assess the breadth of tools available (Sarma, Redmiles, \& Van der Hoek, 2010), adapt to new technologies as they become available (Majchrzak, Rice, Malhotra, King, \& Ba, 2000), and grow in the understanding of sharing knowledge as a normative means by which to conduct open science. These issues will test the control/trust dynamic and decision-making capabilities of individuals in teams to navigate the sharing of their knowledge within the network.

\section{GROUP-DRIVEN TO USER-DRIVEN FUNCTIONALITY AND ORGANIZING}

It is not uncommon for team members and/or stakeholders to display variability or inequity in skill sets necessary for collaboration. In fact, the desire to access scientific skills that are not totally available to any one investigator or even a specific group motivates many teams and stakeholders to collaborate (Beaver \& Rosen, 1979; Hara, Solomon, Kim, \& Sonnenwald, 2003; Melin, 2000; Nash, 2008; Wray, 2006). These abilities having to do with disciplinary expertise, experience with specific methodologies, and even access to instrumentation, data or populations, as they relate to abilities to utilize and maximize the usefulness of technological tools, vary within groups. However, in addition to these scientific skills, a series of soft skills are also necessary for scientific success (Bennett, Maton, \& Kervin, 2008; Gallagher et al., 2005; Mairesse, Greenan, \& Topiol-Bensaid, 2001). 
In open science systems, requisite abilities to utilize and master core technological functionality in machine-assisted work is a strong indicator of the impact technologies can have on the success of a team's contribution to open science. In order for technology to be applied productively, a user must be able to understand both its function and its relationships with other related technologies. In the case of open science technologies, quality of science and consumer's scientific understanding can be dependent on the capacity of users.

User-driven access control, or the freedom to decide to what degree and extent one might share one's work with the network, like functionalities available in the OSF, provides a dimension to requisite teaming skills. This is an opportunity for a departure from the confines of group-decision based knowledge sharing that can be the result of compromises or consensus about how knowledge is or should be integrated (Roesner et al., 2012). Dissemination of knowledge, even within the most collaborative teams, depends on decision-making processes that do not strive to meet all of the needs of individual team members. Publications, reports, or even co-authored electronic entities are subject to an internal polity that can lack a reflection of individual team members' particular or preferred contributions. This can leave individuals subject to decision-making technicalities that result in their work being blended and integrated with others' with more-or-less emphasis on each team member's needed recognition. Though usually an important and positive result of the collaborative process, many of the ownership and authorship challenges described already can serve as mechanisms by which individuals can feel underrepresented, thus, threatening their autonomy. These threats can affect an individual's own willingness to share and the quality of the team's work as a whole. Userdriven access control, and a recorded activity feed or version control contributor record, allow for individual contributions to be identified within the context of group publication and scientific exchange. Such a record alleviates, at least in some cases, recognition concerns for individuals in teams who experience such threats to their contributions.

The shifting from group to user functionality in an open science network is similar to shifts between group-organizing to self-organizing. As with the previous dynamic, this shift may seem antithetical to the point of collaborative engagement. With similar challenges to those that impede an individual's ability to make decisions free from the group, as in user driven functionality when using communication technologies, here the emphasis is on individuals' ability to organize information based on their own decision processes independently yet congruently with the team working on similar problems (Leydesdorff, 2001). For example, any member of a team using the OSF, with read/write or administrative privileges, can alter the display order of contributor names on any project components with or without the consensus of the team. Thus, each member has self-organizing agency. Individuals must choose to work as a team instead of the tool's structure requiring and enforcing a hierarchical team structure.

Though we often think of shared communication spaces (the web, internet, shared drives, social media, etc.) as decentralized and freeform spaces in which users have ultimate autonomy, control, and decision-making authority, these spaces depend greatly on self-organization, the ability to natigate and mevuever amidst changing landscapes, as an important function of the collective knowledge that is integrated and presented as cumulative knowledge. Technologies that capitalize on the userindependence from the network and enhanced user-driven functionality positively impact network outcomes as communities of knowledge are more fluidly accessible to one another (Flake, Lawrence, Giles, \& Coetzee, 2002). Such fluid exchanges permit both similar and divergent communities to interact in ways that would be unlikely using more traditional venues. This fluidity of interaction may increase the likelihood of advancing science while it is ongoing rather than only after it concludes This can allow for teams working on certain problems to interface with other teams working on adjacent problems and enter into dialogue that allows for various levels of knowledge sharing to be conducted simultaneously across different subunits in different phases of research. 


\section{CONCLUSION}

The intention of this paper is to introduce a conversation about how team scholarship and the collaborative tools that are available to modern scholars shed light on a new era of knowledge generation and sharing. As is always the case, technology far out-paces the human capacity to fully utilize and master it. As this current information age progresses, humans will continue to be challenged as to how to harness the power that these constantly advancing technologies provide while evolving with it. Scientific teams, their stakeholders, and the consumers of the science they generate can be brought closer together through machine-assisted communication. In many cases this will accelerate science and its impact on society (Evans \& Reimer, 2009; Gargouri et al., 2010). In some cases, the mere power of available technologies will hamper our willingness to become deeply sharing scholars; "sharing" being a paradigm that still has trouble taking root in our academic and scholarly communities.

The adoption of machine-assisted scholarly activity depends on tangible structures like access to useful technologies and the infrastructures necessary to support them. Over time, with the increased availability of tools and frameworks, adoption of open science infrastructure is becoming more normative. However, as the barriers to access diminish, time will tell if cultural barriers that are rooted in individual reluctance to share knowledge, concerns about receiving or distributing recognition and reward for new forms of scholarly contribution, and negative attitudes about transparency will diminish as well. The group behaviors that embrace open science need to be encouraged by team science leaders and exercised by team members and stakeholders so that the sharing of knowledge becomes paramount over some of the more secondary barriers found in the human condition that can stifle real scientific advancement.

Ultimately, the advancement of open science relies on trust and communication, which are far from new concerns. In fact, many organizational and psychological scientists would report that all effectiveness in teams is grounded in some form (functions and dysfunctions) of trust and the abilities of teams to communicate effectively. Here we provide a mediator of trust and communication found through the application of machine-assisted open science. The landscape of this emerging paradigm carries with it a means in which to advance science through multilevel and cross-sector communication. This in turn advances the collective abilities of scientific teams: their ability to remain in an intimate relationship with their stakeholders and to maximize their impact on the communities that their science hopes to improve.

\section{REFERENCES}

Andriessen, J. (2006). To share or not to share, that is the question: Conditions for the willingness to share knowledge. Delft Innovation System Papers, (IS - 2006-02). Delft, The Netherlands.

Alicea, B. (2016). Data reuse as a prisoner's dilemma: The social capital of open science. BioRxiv. https://doi.org/10.1101/093518

Armstrong, A., \& Jackson-Smith, D. (2013). Forms and levels of integration: Evaluation of an interdisciplinary team-building project. Journal of Research Practice, 9(1), M1.

ArXives of earth science. (2018). Nature Geoscience, 11(3), 149. https://doi.org/10.1038/s41561-018-0083-y

Assante, M., Candela, L., Castelli, D., Manghi, P., \& Pagano, P. (2015). Science 2.0 Repositories: Time for a Change in Scholarly Communication. D-Lib Magazine, 21(1/2). https://doi.org/10.1045/january2015$\underline{\text { assante }}$

Bahney, C., Bruder, S., Cain, J., Keyak, J., Killian, M., Shapiro, I., \& Jones, L. (2016). Accelerating the pace of discovery in orthopaedic research: A vision toward team science. Journal of Orthopaedic Research, 34(10), 1673-1679. https://doi.org/10.1002/jor.23307 
Bales, M., Dine, D., Merrill, J. A., Johnson, S., Bakken, S., \& Weng, C. (2014). Associating co-authorship patterns with publications in high-impact journals. Journal of Biomedical Informatics, 52, 311-318. https://doi.org/10.1016/j.jbi.2014.07.015

Barrett, K., Funk, C., \& Macrina, F. (2005). Awareness of publication guidelines and the responsible conduct of research. Accounting Research, 12(3), 193-206. https://doi.org/10.1080/08989620500217321

Beaver, D., \& Rosen, R. (1979). Studies in scientific collaboration Part III: Professionalism and the natural history of modern scientific co-authorship. Scientometrics, 1(3), 231-245. https://doi.org/10.1007/BF02016308

Bennet, L. M., Gadlin, H., \& Levine-Finley, S. (2010). Collaboration and team science: A field guide. Bethesda, MD: National Institutes of Health.

Bennett, S., Maton, K. A., \& Kervin, L. (2008). The 'digital natives' debate: A critical review of the evidence. British Journal of Educational Technology, 39(5), 775-786. https://doi.org/10.1111/j.1467-8535.2007.00793.x

Berg, J., Bhalla, N., Bourne, P., Chalfie, M., Drubin, D., Fraser, J., . . Wolberger, C. (2016). Preprints for the life sciences. Science, 352(6288), 899-901. https://doi.org/10.1126/science.aaf9133

Bock, G-W, Zmud, R. W., Kim, Y-G, \& Lee, J-N. (2005) Behavioral intention formation in knowledge sharing: Examining the roles of extrinsic motivators, social-psychological forces, and organizational climate, MIS Quarterly 29(1), 87-111. https://doi.org/10.2307/25148669

Brick, C., \& Regenhardt, C. (n.d.). About the Project, Eleanor Roosevelt Papers Project. Retrieved from https://erpapers.columbian.gwu.edu/about-project

Burke, C., Stagl, K., Klein, C., Goodwin, G., Salas, E., \& Halpin, S. (2006). What type of leadership behaviors are functional in teams? A meta analysis. Leadership Quarterly, 17, 288-307. https://doi.org/10.1016/j.leaqua.2006.02.007

Carayol, N., \& Thi, T. (2005). Why do academic scientists engage in interdisciplinary research? Research Evaluation, 14(1), 70-79. https://doi.org/10.3152/147154405781776355

Center for Open Science. (n.d.-a). Registered reports. Retrieved March 20, 2018, from https://cos.io/rr/

Center for Open Science. (n.d.-b). The Open Science Framework. Retrieved March 20, 2018, from https://cos.io/our-products/osf/

Coleman, J. (1986). Social theory, social research, and a theory of action. The American Journal of Sociology, 91(6), 1309-1335. https://doi.org/10.1086/228423

Dabbish, L., Stuart, C., Tsay, J., \& Herbsleb, J. (2012). Social coding in GitHub: Transparency and collaboration in an open software repository. In Proceedings of the ACM 2012 Conference on Computer Supported Cooperative Work (pp. 1277-1286). New York, NY, USA: ACM. https://doi.org/10.1145/2145204.2145396

Dasgupta, P., \& David, P. (1994). Toward a new economics of science. Policy Research, 23, 487-521. https://doi.org/10.1016/0048-7333(94)01002-1

Drath, W., McCauley, C., Palus, C., Van Velsor, E., O’Connor, P., \& McGuire, J. (2008). Direction, alignment, commitment: Toward a more integrative ontology of leadership. Leadership Quarterly, 19(6), 635-653. https://doi.org/10.1016/j.leaqua.2008.09.003

Engwall, L., \& Blockmans, W. (2014). Bibliometrics: Use and abuse in the review of research performance. London: Portland Press.

Evans, J. A., \& Reimer, J. (2009). Open access and global participation in science. Science, 323(5917), 1025. https://doi.org/10.1126/science.1154562

Fecher, B., \& Friesike, S. (2014). Open science: One term, five schools of thought. In S. Bartling \& S. Friesike (Eds.), Opening science - The evolving guide on how the web is changing research (pp. 17-47). Springer, Cham. https://doi.org/10.1007/978-3-319-00026-8 2

Fister, B. (2016, August 18). The acceleration of open access. Retrieved March 8, 2018, from https://www.insidehighered.com/blogs/library-babel-fish/acceleration-open-access 
Flake, G., Lawrence, S., Giles, C., \& Coetzee, F. (2002). Self-organization and identification of web communities. IEEE Computer Supported Cooperative Work, 35(3), 66-71. https://doi.org/10.1109/2.989932

FOSTER Plus. (2016). Open science definition. Retrieved from https://www.fosteropenscience.eu/fostertaxonomy/open-science-definition

Fox, M., \& Faver, C. (1984). Independence and cooperation in research: The motivations and costs of collaboration. The Journal of Higher Education, 55(3), 347-359. https://doi.org/10.1080/00221546.1984.11777069

Gallagher, A., Ritter, E., Champion, H., Higgins, G., Fried, M., Moses, G., . . Satava, R. (2005). Virtual reality simulation for the operating room: Proficiency-based training as a paradigm shift in surgical skills training. Annals of Surgery, 241(2), 364-372. https://doi.org/10.1097/01.sla.0000151982.85062.80

Gargouri, Y., Hajjem, C., Larivière, V., Gingras, Y., Carr, L., Brody, T., \& Harnad, S. (2010). Self-selected or mandated, open access increases citation impact for higher quality research. PLOS ONE, 5(10), e13636. https://doi.org/10.1371/journal.pone.0013636

Georghiou, L. (1988). Global cooperation in research. Research Policy, 27(6), 611-626. https://doi.org/10.1016/S0048-7333(98)00054-7

Gibson, K. (2000). The moral basis of stakeholder theory. Journal of Business Ethics, 26(3), 245-257. https://doi.org/10.1023/A:1006110106408

Gravani, M. (2005). Academics and practitioners: partners in generating knowledge or citizens of two different worlds? Paper presented at the European Conference on Educational Research, Dublin.

Hall, K., Feng, A., Moser, R., Stokols, D., \& Taylor, B. (2008). Moving the science of team science forward: Collaboration and creativity. American Journal of Community Psychology, 35(supplement), S243-249. https://doi.org/10.1016/j.amepre.2008.05.007

Hall, K. L., Stokols, D., Stipelman, B. A., Vogel, A. L., Feng, A., Masimore, B., .. Berrigan, D. (2012). Assessing the value of team science: A study comparing center- and investigator-initiated grants. American Journal of Preventive Medicine, 42(2), 157-163. https://doi.org/10.1016/j.amepre.2011.10.011

Hansen, S., \& Avital, M. (2005). Share and share alike: The social and technological influences on knowledge sharing behavior. Sprouts: Working Papers on Information Systems, 5(13).

Hara, N., Solomon, P., Kim, S.-L., \& Sonnenwald, D. (2003). An emerging view of scientific collaboration: Scientists' perspectives on collaboration and factors that impact collaboration. Journal of the American Society for Information Science Technology, 54(10), 952-965. https://doi.org/10.1002/asi.10291

Harris, F., Lyon, F., \& Clarke, S. (2009). Doing interdisciplinarity: Motivation and collaboration in research for sustainable agriculture in the UK. Area, 41(4), 374-384. https://doi.org/10.1111/j.1475-4762.2008.00859.x

Haythornthwaite, C., Lunsford, K., Bowker, G., \& Bruce, B. (2006). Challenges for research and practice in distributed, interdisciplinary collaboration. In C. Hine (Ed.), New infrastructures for knowledge production: Understanding E-science (pp. 143-166). https://doi.org/10.4018/978-1-59140-717-1.ch007

Hendriks, P. (1999). Why share knowledge. Knowledge and Process Management, 6(2), 91-100. https://doi.org/10.1002/(SICI)1099-1441(199906)6:2<91::AID-KPM54>3.0.CO;2-M

Hinnant, C., Stvilia, B., Wu, S., Worrall, A., Burnett, G., Burnett, K., . . Marty, P. (2012). Author team diversity and the impact of scientific publications: Evidence from physics research at a national science lab. Library \&Information Science Research, 34(4), 249-257. https://doi.org/10.1016/j.lisr.2012.03.001

Hoffman, R., Ward, P., Feltovich, P., DiBello, L., Fiore, S., \& Andncyrews, D. (2013). Accelerated expertise: Training for high proficiency in a complex world. East Sussex: Psychology Press.

Holdren, J. (2013). Increasing access to the results of federally funded scientific research. Retrieved from https://obamawhitehouse.archives.gov/sites/default/files/microsites/ostp/ostp public access memo 2 013.pdf

Horlick-Jones, T., \& Sime, J. (2004). Living on the border: Knowledge, risk and transdisciplinarity. Futures, 36 , 441-456. https://doi.org/10.1016/i.futures.2003.10.006 
Hower, M. (2012). Faculty work: Moving beyond the paradox of autonomy and collaboration. (PhD), Antioch University.

Inter-university Consortium for Political and Social Research. (n.d.). About ICPSR. Retrieved from https://www.icpsr.umich.edu/icpsrweb/content/about/

International DOI Foundation. (n.d.). Factsheet. DOI system and the Handle System. Retrieved from http://www.doi.org/factsheets/DOIHandle.html

Johnson, M. (2017, November 27). GenBank Overview. Retrieved April 30, 2018, from https://www.ncbi.nlm.nih.gov/genbank/

Jones, B., Wuchty, S., \& Uzzi, B. (2008). Multi-university research teams: Shifting impact, geography, and stratification in science. Science, 322, 1259-1262. https://doi.org/10.1126/science.1158357

Klein, C., DeRouin, R., \& Salas, E. (2006). Uncovering workplace interpersonal skills: A review, framework, and research agenda. In G. Hodgkinson \& J. Ford (Eds.), International review of industrial and organizational psychology (Vol. 21, pp. 80-126). New York: John Wiley and Sons. https://doi.org/10.1002/9780470696378.ch3

Klein, W., \& Bloom, M. (2005). Bibliometrics: The best available information? Journal Social Work in Health Care, 41(3/4). https://doi.org/10.1300/J010v41n03 07

Kosara, R., Hauser, H., \& Gresh, D. L. (2003). An interaction view on information visualization. Proceedings of EUROGRAPHICS, 123-137.

Kozlowski, S., Watola, D., Jensen, J., Kim, B., \& Botero, I. (2009). Developing adaptive teams: A theory of dynamic team leadership. In E. Slas, G. Goodwin, \& C. Burke (Eds.), Team effectiveness in complex organizations: Cross disciplinary perspectives and approaches (pp. 113-155). New York: Routledge.

LabArchives. (2018, March 15). 6.2 Other ways to Share (URLs, DOIs). Retrieved March 20, 2018, from http://labarchives.kayako.com/Knowledgebase/Article/View/142/225/62-other-ways-to-share-urls-dois

Lewis, J., Ross, S., \& Holden, T. (2012). The how and why of academic collaboration: Disciplinary differences and policy implications. Higher Education, 64(5), 693-708. https://doi.org/10.1007/s10734-012-9521-8

Leydesdorff, L. (2001). A sociological theory of communication: The Self-organization of the knowledge-based society. Universal-Publishers

Liljander, V., Gillberg, F., Gummerus, J., \& van Riel, A. (2006). Technology readiness and the evaluation and adoption of self-service technologies. Journal of Retailing and Consumer Services, 13, 177-191.

https://doi.org/10.1016/i.jretconser.2005.08.004

Lin, C.-H., Shih, H.-U., \& Sher, P. (2007). Integrating technology readiness into technology acceptance: The TRAM Model. Psychology and Marketing, 24(7), 641-657. https://doi.org/10.1002/mar.20177

Lotrecchiano, G., Mallinson, T., Leblanc-Beaudoin, T., Schwartz, L., Lazar, D., \& Falk-Krzesinski, H. (2016). Motivation and threat indicators for collaboration readiness in knowledge generating teams (KPTs): A scoping review and domain analysis. Heliyon, 2(5), e00105. https://doi.org/10.1016/j.heliyon.2016.e00105

Maglaughlin, K., \& Sonnenwald, D. (2005). Factors that impact interdisciplinary natural science research collaboration in academia. Paper presented at the International Society for Scientometrics and Informetrics (ISSI).

Mairesse, J., Greenan, N., \& Topiol-Bensaid, A. (2001). Information technology and research and development impacts on productivity and skills: Looking for correlations on French firm level data. National Bureau of Economic Research Working Paper No. 8075. https://doi.org/10.3386/w8075

Majchrzak, A., Rice, R., Malhotra, A., King, N., \& Ba, S. (2000). Technology adaptation: The case of a computer-supported inter-organizational virtual team. MIS Quarterly, 24(4), 569-600. https://doi.org/10.2307/3250948

Mallinson, T., Lotrecchiano, G., Furniss, J., Schwartz, L., Lazar, D., \& Falk-Krzesinski, H. (2016). Rasch Analysis as a method for designing a readiness model for collaboration. Journal of Investigative Medicine, 64(7), 1186-1193. https://doi.org/10.1136/jim-2016-000173

McDermott, R., \& O’Dell, C. (2001). Overcoming cultural barriers to sharing knowledge. Journal of Knowledge Management, 5(5), 76-85. https://doi.org/10.1108/13673270110384428 
Melin, G. (2000). Pragmatism and self-organization: Research collaboration on the individual level. Research Policy, 29(1), 31-40. https://doi.org/10.1016/S0048-7333(99)00031-1

Misra, S., Stokols, D., \& Cheng, L. (2015). The transdisciplinary orientation scale: Factor structure and relation to the integrative quality and scope of scientific publications. Journal of Translational Medicine and Epidemiology, 3(2), 1042.

Nash, J. (2008). Transdisciplinary training: Key components and prerequisites for success. American Journal of Preventive Medicine, 35(2S), 133-140. https://doi.org/10.1016/i.amepre.2008.05.004

National Research Council. (2015). Enhancing the effectiveness of team science. (N. J. Cooke \& M. L. Hilton, Eds.). Washington, DC: The National Academies Press. https://doi.org/10.17226/19007

Nosek, B., Alter, G., Banks, G., Borsboom, D., Bowman, S., Breckler, S., . . Yarkoni, T. (2015). Promoting an open research culture. Science, 348(6242), 1422-1425. https://doi.org/10.1126/science.aab2374

Obama, B. (2015). Using behavioral science insights to better serve the American people [Press release]. Retrieved from https://obamawhitehouse.archives.gov/the-press-office/2015/09/15/executive-order-using-behavioralscience-insights-better-serve-american

Olson, G. M., \& Olson, J. S. (2000). Distance matters. Human-Computer Interaction, 15, 139-178. https://doi.org/10.1207/S15327051HCI1523 4

Parasuraman, R., Sheridan, T., \& Wickens, C. (2000). A model for types and levels of human interaction with automation. IEEE Transactions on Systems, Man, and Cybernetics, 30(3), 286-297. https://doi.org/10.1109/3468.844354

Pennington, D. (2011). Bridging the disciplinary divide: Co-creating research ideas in eScience teams. Computer Supported Cooperative Work, 20(3), 165-196. https://doi.org/10.1007/s10606-011-9134-2

Porter, A., Roessner, J., \& Heberger, A. (2008). How interdisciplinary is a given body of research? Research Evaluation, 17(4), 273-282. https://doi.org/10.3152/095820208X364553

Priem, J., Taraborelli, D., Groth, P., \& Neylon, C. (2010). Altmetrics: A manifesto (v 1.01). Retrieved from http://altmetrics.org/manifesto/

Ranwala, D., Alberg, A., Brady, J., Obeid, J., Davis, R., \& Halushka, P. (2017). Scientific retreats with 'speed dating': Networking to stimulate new interdisciplinary translational research collaborations and team science. Journal of Investigative Medicine, 65(2), 382-390. https://doi.org/10.1136/jim-2016-000261

Rechberg, I., \& Syed, J. (2013). Ethical issues in knowledge management: Conflict of knowledge ownership. Journal of Knowledge Management, 17(6). https://doi.org/10.1108/JKM-06-2013-0232

Rhoten, D., \& Parker, A. (2004). Education: Risks and rewards of an interdisciplinary research path. Science, 306 , 2046. https://doi.org/10.1126/science.1103628

Roesner, F., Tadayoshi, K., Moshchuk, A., Parno, B., Wang, H., \& Cowan, C. (2012). User-driven access control: Rethinking permission granting in modern operating systems. Paper presented at the Proceedings of the IEEE Symposium on Security and Privacy. https://doi.org/10.1109/SP.2012.24

Rollman, J., Krug, K., \& Parente, F. (2004). The chat room phenomenon: Reciprocal communication in cyberspace. CyberPsychology \& Behavior, 3(2), 161-166. https://doi.org/10.1089/109493100316003

Rosas, S., Kagan, J., Schouten, J., Slack, P., \& Trochim, W. (2011). Evaluating research and impact: A bibliometric analysis of research by the NIH/NIAID HIV/AIDS clinical trials networks. PLoS ONE, 6(3), e17428. https://doi.org/10.1371/journal.pone.0017428

Salazar, M., Lant, T., Fiore, S., \& Salas, E. (2012). Facilitating innovation in diverse science teams through integrative capacity. Small Group Research, 43(5), 527-558. https://doi.org/10.1177/1046496412453622

Sarma, A., Redmiles, D., \& Van der Hoek, A. (2010). Categorizing the spectrum of coordination technology. IEEE Computer Supported Cooperative Work, 43(6), 61-67. https://doi.org/10.1109/MC.2010.163

Scheliga, K., \& Friesike, S. (2014). Putting open science into practice: A social dilemma? First Monday, 19(9). https://doi.org/10.5210/fm.v19i9.5381 
Sonnenwald, D. H. (2007). Scientific collaborations: A synthesis of challenges and strategies. In B. Cronin (Ed.), Annual review of information sciences and technology (Vol. 41). Medford, NJ: Information Today, Inc. https://doi.org/10.1002/aris.2007.1440410121

Spiegler, I. (2003). Technology and knowledge: Bridging a "generating" gap. Information and Management, 40(6), 533-539. https://doi.org/10.1016/S0378-7206(02)00069-1

Srivastava, A., Bartol, K., \& Locke, E. (2006). Empowering leadership in management teams: Effects on knowledge sharing, efficacy, and performance. The Academy of Management Review, 49(6), 1239-1251. https://doi.org/10.5465/AMJ.2006.23478718

Stokols, D. (2014). Training the next generation of transdisciplinarians. In M. O'Rourke, S. Crowley, S. D. Eigenbrode, \& J. D. Wulfhorst (Eds.), Enhancing communication \& collaboration in interdisciplinary research (pp. 56-81). Los Angeles, CA: Sage Publication. https://doi.org/10.4135/9781483352947.n4

Stokols, D., Hall, K., Taylor, B., \& Moser, R. (2008). The science of team science: Overview of the field and introduction to the supplement. American Journal of Preventative Medicine, 35 (2 Supplement) (S77-S89). https://doi.org/10.1016/j.amepre.2008.05.002

Suber, P. (2012). MIT press essential knowledge: Open access. Cambridge, MA., United States: MIT Press. Retrieved from http://ebookcentral.proquest.com/lib/gwu/detail.action?docID=3339454

Swift, M., Balkin, D., \& Matusik, S. (2010). Goal orientations and the motivation to share knowledge. Journal of Knowledge Management, 14(3), 378-393. https://doi.org/10.1108/13673271011050111

Tannenbaum, S., Mathieu, J., Salas, E., \& Cohen, D. (2012). Teams are changing: Are research and practice evolving fast enough? Industrial and Organizational Psychology, 5(1), 2-24. https://doi.org/10.1111/j.17549434.2011.01396.x

Turpin, T., \& Garrett-Jones, S. (2010). Reward, risk and response in Australian cooperative research centres. International Journal of Technology Transfer and Commercialization, 9(1-2), 77-93. https://doi.org/10.1504/IJTTC.2010.029426

Walczuch, R., Lemmink, J., \& Streukens, S. (2007). The effect of service employees' technology readiness on technology acceptance. Information and Management, 44, 206-215. https://doi.org/10.1016/j.im.2006.12.005

Weimer, K., \& Andrew, P. (2013). How we participate in the scholarly communication life cycle. Journal of Map \& Geography Libraries: Advances in Geospatial Information, Collections \& Archives, 9(3), 217-219. https://doi.org/10.1080/15420353.2013.824397

Wray, K. (2006). Scientific authorship in the age of collaborative research. Studies in History and Philosophy of Science Part A, 37(3), 505-514. https://doi.org/10.1016/j.shpsa.2005.07.011

Wuchty, S., Jones, B., \& Uzzi, B. (2007). The increasing dominance of teams in production of knowledge. Science, 316, 1036-1038. https://doi.org/10.1126/science.1136099

Zucker, D. (2012). Developing your career in an age of team science. Journal of Investigative Medicine, 60(5), 779784. https://doi.org/10.2310/JIM.0b013e3182508317

\section{BIOGRAPHIES}

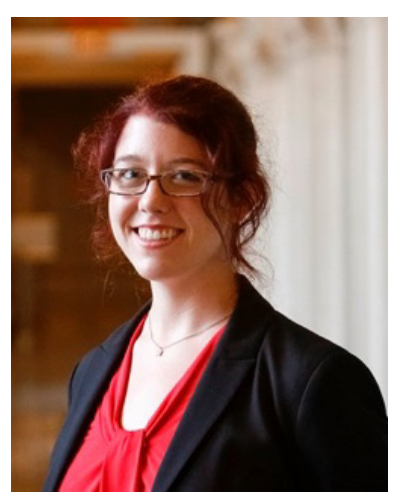

Megan Potterbusch is the data services librarian for George Washington University. She works with faculty and student researchers on data access and research data management and has a particular interest in the theory and practice of open science. Potterbusch received her masters of library and information science from Simmons College, and was a National Digital Stewardship Resident focused on implementing open science and digital stewardship principles through outreach and supporting researchers to explore new multi-function tools and technology. 


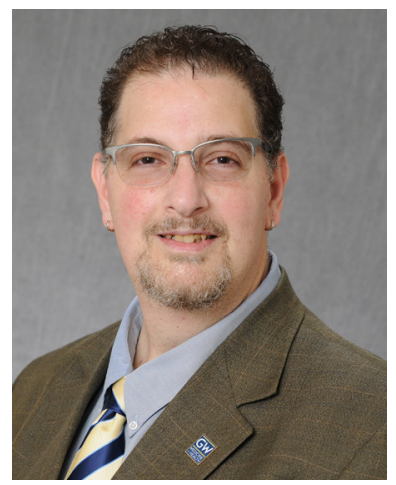

Gaetano R. Lotrecchiano, EdD, PhD is an Associate Professor at the George Washington University School of Medicine and Health Sciences, Washington, DC USA where he is the Director of Doctoral Candidacy in the $\mathrm{PhD}$ in Translational Health Sciences Program. He is the foundation vice-president of the International Society for Systems and Complexity Sciences for Health and of the International Society of the Science of Team Science. He is the convener of the GWU program entitled Creating a Culture of Collaboration at GWU. He is also the Team Science Lead of the Clinical and Translational Science Institute (CTSI-CN), a partnership between Children's National Health System and George Washington University. 